\title{
INDUCTION OF TOLERANCE TO AN EXPERIMENTAL CARDIAC ALLOGRAFT THROUGH INTRATHYMIC INOCULATION OF CLASS II MAJOR HISTOCOMPATIBILITY COMPLEX DISPARATE ANTIGENS
}

Zhenya Shen, MD

Muhammad Mohiuddin, MD

Hitoshi Yokoyama, MD, PhD

G. Russell Reiss, MD

Verdi J. DiSesa, MD
Indefinite donor-specific tolerance to a cardiac allograft can be induced through pretransplantation intrathymic injection of donor spleen cells and a single intraperitoneal injection of antilymphocyte serum. This study was designed to determine whether this phenomenon was reproducible with grafts differing in either class I major histocompatibility complex only or class II MHC only. Donors of cells and hearts in all experiments were RP rats. Class I MHC disparate grafts were performed by placing an RP heart into a Lewis recipient, and class II disparate grafts were performed with RP donors and Wistar Furth recipients. Lewis $(n=10)$ and Wistar Furth $(n=$ 10) recipients underwent intraperitoneal injection of $1 \mathrm{ml}$ antilympocyte serum and intrathymic injection of $5 \times 10^{7}$ RP spleen cells. Three weeks later, heterotopic cardiac transplantation was done with a heart from an RP rat. Control rats had no pretreatment or received antilympocyte serum alone. Without pretreatment, RP hearts survived 7 to 9 days (mean 8 days) in Lewis recipients $(n=5)$ and 9 to 14 days (mean 12 days) in Wistar Furth recipients $(n=5)$. Antilymphocyte serum alone produced slight prolongation of graft survival. Lewis rats pretreated with class I disparate RP splenocytes and antilympocyte serum had graft survivals of 8 to 27 days (mean 14 days), not significantly different from the results with antilympocyte serum alone. Class II disparate RP grafts placed in pretreated Wistar Furth rats had significant prolongation of graft survival, with four of five grafts surviving longer than 60 days $(p<0.01$ vs antilympocyte serum alone). These results suggest that a disparity at the class II locus of the major histocompatibility complex is critical for the induction of cardiac allograft tolerance after intrathymic inoculation of allogeneic cells. (J Thorac Cardiovasc Surg 1996;112:1315-8)
Induction of donor-specific unresponsiveness represents an ideal strategy through which survival of tissue or organ allografts could be promoted without chronic host immunosuppression. Indefinite donor-

From the Department of Cardiothoracic Surgery, The Medical College of Pennsylvania and Hahnemann University School of Medicine, Philadelphia, Pa.

Read at the Seventy-sixth Annual Meeting of The American Association for Thoracic Surgery, San Diego, Calif., April 28-May 1, 1996.

Received for publication March 25, 1996; revisions requested May 8, 1996; revisions received May 17, 1996; accepted for publication May 21, 1996.

Address for reprints: Verdi J. DiSesa, M.D., Cardiothoracic Surgery, Medical College of Pennsylvania Hospital, 3300 Henry Avenue, Philadelphia, PA 19129.

Copyright (c) 1996 by Mosby-Year Book, Inc.

$0022-5223 / 96 \$ 5.00+0 \quad \mathbf{1 2 / 6 / 7 5 2 0 0}$ specific tolerance to a cardiac allograft disparate in both class I and class II major histocompatibility complex (MHC) antigens was achieved in our laboratory and others through the pretransplant intrathymic injection of donor spleen cells and a single intraperitoneal injection of antilymphocyte serum (ALS). ${ }^{1-4}$ The underlying mechanism of tolerance in this model has not yet been defined. It is commonly believed that a form of self-tolerance induced in the thymus is altered by the injection of cells expressing foreign antigens. Recently, several experiments have addressed the issue of whether donor MHC alloantigens are necessary for the intrathymic induction of tolerance. ${ }^{5-7}$ In previous experiments, we showed that the efficacy in prolonging cardiac allograft survival of intrathymic pretreatment with allogeneic cells is related to the degree of donor-recipient histoincompatibility. ${ }^{4}$ This study, which is an exten- 
Table I. Survival of cardiac allografts in MHC mismatched recipients pretreated by intrathymic injection with donor allogeneic antigens

\begin{tabular}{|c|c|c|c|c|c|c|c|}
\hline \multirow[b]{2}{*}{ Group } & \multirow[b]{2}{*}{ Donor } & \multirow[b]{2}{*}{ Recipient } & \multirow[b]{2}{*}{ MHC mismatch } & \multirow[b]{2}{*}{ Pretreatment } & \multicolumn{2}{|c|}{ Survival (days) } & \multirow[b]{2}{*}{$p^{*}$} \\
\hline & & & & & Raw values & Mean & \\
\hline \multirow[t]{2}{*}{$\mathrm{a}$} & $\mathrm{RP}$ & Lewis & Class I & None & $7,7,9,9,9$ & 8.2 & - \\
\hline & $\mathrm{RP}$ & WF & Class II & None & $9,11,13,14,14$ & 12.2 & - \\
\hline \multirow[t]{2}{*}{$b$} & $\mathrm{RP}$ & Lewis & Class I & IP ALS & $9,10,13,13,14$ & 11.8 & $<0.05 \dagger$ \\
\hline & RP & WF & Class II & IP ALS & $13,14,14,15,15$ & 14.2 & $\mathrm{NS}+$ \\
\hline \multirow[t]{2}{*}{$\mathrm{c}$} & $\mathrm{RP}$ & Lewis & Class I & IT RPSC & $8,10,11,13,27$ & 13.8 & NSł \\
\hline & $\mathrm{RP}$ & WF & Class II & IT RPSC & $19,>60,>60,>60,>60$ & 51.8 & $<0.01 \neq$ \\
\hline
\end{tabular}

NS, not significant; RPSC, splenocytes from RP rat.

*Unpaired two-tailed $t$ test.

†Versus group a.

\$ersus groups a and $\mathrm{b}$.

sion of our investigations on histocompatibility differences and cardiac transplant tolerance produced by intrathymic pretreatment, was designed to determine whether the donor-specific tolerance produced by intrathymic pretreatment was reproducible with grafts differing in either class I MHC only or class II MHC only.

\section{Materials and methods}

Animals. The rat MHC (RT1) is subdivided into five loci $(\mathrm{A}, \mathrm{B}, \mathrm{C}, \mathrm{D}$, and $\mathrm{E}){ }^{8}$ The rat RT1A and RT1C regions encode class I (major) and class I (medial) transplantation antigens, respectively. The RT1B and RT1D regions encode class II transplantation antigens. RP (RT1 AuBlDl), Lewis (RT1 AlBIDlCl), and Wistar Furth (WF; RT1 AuBuDuCu) rats were used in these experiments. The RP and Lewis strains have class I MHC histoincompatibilities, whereas the RP and WF strains are class II MHC histoincompatible. All rats studied were male adults (200 to $250 \mathrm{gm}$ ). RP rats were bred at the Central Animal Facilities of Erasmus University (Rijswijk, The Netherlands). The other strains were obtained from Harlan Sprague Dawley, Inc. (Indianapolis, Ind.). All animals were kept in a virus-free facility at the Medical College of Pennsylvania Hospital and were handled in a humane fashion in accord with Medical Research and the "Guide for the Care and Use of Laboratory Animals" prepared by the Institute of Laboratory Animal Resources and published by the National Institutes of Health (NIH Publication No. 86-23, revised 1985).

Experimental design. Donors of cells and hearts in all experiments were RP rats. Grafts were done with the following strain combinations and across the following histoincompatibility lines: class I MHC, RP into Lewis; class II MHC, RP into WF. Recipient animals were divided into three subgroups: group a, no pretreatment; group $b$, intraperitoneal injection of ALS only; and group c, ALS and intrathymic pretreatment with splenocytes from RP donors.

Preparation of donor spleen cells. Whole RP spleens were removed through a midline incision. Spleens were disrupted by use of a tissue homogenizer and suspended in RPMI 1640 medium (Sigma Chemical Co., St. Louis, Mo.). Red blood cells were lysed with ammonium chloride. Remaining white blood cells were washed with RPMI 1640 medium. Viability of the cells was evaluated by trypan blue dye exclusion.

Pretreatment of graft recipients. On the day before intrathymic treatment, recipient rats received $1 \mathrm{ml}$ ALS intraperitoneally. The next day, after metofane inhalation anesthesia was induced, the thymus of the recipient rat was exposed by a partial median sternotomy and a dose of $2.5 \times 10^{7}$ donor splenocytes in a volume of $0.05 \mathrm{ml}$ was injected into both lobes of the thymus under direct vision.

Heart transplantation. Three weeks after pretreatment or injection of ALS alone, recipient animals underwent transplantation under metofane anesthesia with hearts from donors of the same strains as those used to obtain splenocytes for intrathymic injection. Hearts were transplanted heterotopically in the manner described by Ono and Lindsey. ${ }^{9}$ Graft viability was assessed by daily abdominal palpation; if palpation was indeterminate, viability was assessed by direct visualization. Rejection, marked by the complete absence of ventricular contractions, was confirmed histologically.

Histopathology. The rejected hearts were examined with routine histopathologic staining. Sections of rejected hearts were fixed in $10 \%$ buffered formalin and embedded in paraffin. Staining was carried out with hematoxylin and eosin. Slides were examined by light microscopy.

Statistical analysis. Comparison between groups was done with an unpaired two-tailed Student's $t$ test.

\section{Results}

Heart graft survivals are summarized in Table I. Heart grafts transplanted across either class I MHC or class II MHC mismatch strain combinations were all rejected by untreated recipients; they survived 7 to 9 days (mean 8 days) and 9 to 14 days (mean 12 days), respectively. After administration of ALS only cardiac graft survival was slightly prolonged 
(mean 12 days, $p<0.05$ vs unpretreated group) for Lewis recipients (class I MHC disparate strain combination) but was not prolonged (mean 14 days, $p$ not significant) for WF recipients (class II MHC disparate strain combination).

The results for the two class MHC disparate strain combinations were quite different after intrathymic pretreatment. Graft survival for Lewis rats pretreated with class I disparate RP splenocytes and ALS ranged from 8 to 27 days (mean 14 days), not significantly different from the effect of ALS alone. Class II mismatched RP grafts placed in WF rats after intrathymic pretreatment had significant prolongation of graft survival, with four of five grafts surviving longer than 60 days $(p<0.01$ vs ALS alone).

\section{Discussion}

Previous experiments confirmed the role of the thymus in the induction and maintenance of specific immunologic unresponsiveness to organ allografts. ${ }^{2,5,10}$ One theory hypothesizes that introduction of allogeneic cells into the thymus alters the immunologic milieu in which new $\mathrm{T}$ cells mature. When immature lymphoid cells from the bone marrow circulate through the thymus, their development takes place in the presence of alloantigens presented as "self." In normal immune ontogeny, self-reactive cells are eliminated in the thymus. This process leads to the development of a repertoire of $T$ cells that do not recognize the allogeneic cells in the thymus as foreign. Previous work suggests that the thymic environment can be manipulated to result in the development of donor-specific tolerance across an MHC barrier but not across a non-MHC barrier. ${ }^{41}$ Goss, Nakafusa, and Flye ${ }^{6}$ showed that the form of donor MHC alloantigens placed in the thymus is critical for the induction of donor-specific tolerance and allograft acceptance. This study provides further evidence that specific requirements for antigen presentation in the thymus are quite stringent, even in this rodent model.

Proper presentation of foreign antigens in the thymus is required for tolerance induction by intrathymic pretreatment. This study was designed to test whether class I MHC only or class II MHC only disparate cells were effective in producing cardiac allograft tolerance after intrathymic inoculation. Our results showed that graft tolerance was observed in the class II MHC mismatched strains but not in class I MHC mismatched strains. Class I disparate antigens thus do not appear to be effectively presented in the thymic milieu for the induction of graft tolerance. The reason for this is not clear. It may relate to antigen processing in the thymus or elsewhere, or to the fact that class II antigens, unlike class I MHC antigens, are not expressed constitutively on all somatic cells, especially endothelial cells.

Additionally, our previous studies showed that prolongation of graft survival was longest in strain combinations that differed at the full MHC locus (both class I and class II) after pretreatment with ALS and intrathymic donor alloantigens. These findings suggest that a necessary condition for the induction of tolerance to an experimental cardiac allograft by intrathymic pretreatment is the inoculation of class II MHC disparate antigens.

As in a previous study, ${ }^{4}$ slight prolongation of graft survival was observed in recipients injected with ALS alone. One dose of ALS produces transient reduction in all classes of circulating lymphocytes. The time course of this reduction is such that the recipient animal has not completely regained normal circulating levels of immune competent cells by 3 weeks, the time of engraftment. Presumably, this nonspecific immunosuppressive effect leads to slight prolongation of graft survival in this model.

In summary, our study demonstrates that a disparity at the class II locus of the MHC is critical for the induction of cardiac allograft tolerance after intrathymic inoculation of allogeneic cells. Application of these principles could have implications for clinical transplantation.

\section{REFERENCES}

1. Goss JS, Nakafusa Y, Flye MW. Intrathymic injection of donor alloantigens induces donor-specific vascularized allograft tolerance without immunosuppression. Ann Surg 1992;216:409-16.

2. Kline GM, Shen Z, Mohiuddin MM, et al. Successful experimental heart transplantation without immunosuppressive drugs. J Heart Lung Transplant 1993;12:388-93.

3. Posselt AM, Barker CF, Tomaszewski JE, et al. Induction of donor-specific unresponsiveness by intrathymic islet transplantation. Science 1990;245:1293-5.

4. Shen Z, Kline G, Mohiuddin M, DiSesa VJ. Histocompatibility difference and cardiac transplant tolerance produced by intrathymic pretreatment. J Thorac Cardiovasc Surg 1994; 107:1472-5.

5. Oluwole SF, Chowdhury NC, Fawwaz RA. Induction of donor-specific unresponsiveness to rat cardiac allografts by pretreatment with intrathymic donor MHC class I antigens. Transplantation 1993;55:1396-402. 
6. Goss JA, Nakafusa Y, Flye MW. MHC class II presenting cells are necessary for the induction of intrathymic tolerance. Ann Surg 1993;217:491-501.

7. Oluwole SF, Chowdhury NC, Fawwaz RA, et al. Induction of specific unresponsiveness to rat cardiac allografts by pretreatment with intrathymic donor major histocompatibility complex class I antigens. Transplant Proc 1993;25:299-300.

8. Butcher GW, Howard JC. Handbook of experimental immunology. 3rd ed. London: Blackwell Scientific, 1985;101.1-18.
9. Ono K, Lindsey ES. Improved technique of heart transplantation in rats. J Thorac Cardiovasc Surg 1969;57:225-9.

10. Goss JA, Nakafusa Y, Yu S, Flye MW. Intrathymic injection of donor alloantigens induces specific tolerance to cardiac allografts. Transplantation 1993;56:166-73.

11. Nakafusa Y, Goss JA, Mohanakumaa T, Flye NW. Induction of donor-specific tolerance to cardiac but not skin or renal allografts by intrathymic injection of splenocyte alloantigen. Transplantation 1993;55:887-2. 